\title{
Quality of door sounds of passenger cars
}

\author{
Sonoko Kuwano ${ }^{1, *}$, Hugo Fastl ${ }^{2}$, Seiichiro Namba ${ }^{3}$, Seishi Nakamura ${ }^{4}$ and Hiroshi Uchida ${ }^{4}$ \\ ${ }^{1}$ Graduate School of Human Sciences, Osaka University, \\ 1-2 Yamadaoka, Suita, 565-0871 Japan \\ ${ }^{2}$ Technical University of Munich, \\ Arcisstrasse 21, D-80333 München, Germany \\ ${ }^{3}$ Osaka University \\ ${ }^{4}$ Mazda Motor Co. Ltd., \\ 3-1 Shinchi, Fuchu-cho, Aki-gun, Hiroshima, 730-8670 Japan
}

(Received 3 May 2006, Accepted for publication 12 May 2006)

Keywords: Sound quality, Door sounds of cars, Relation between image of cars and the sounds, German and Japanese participants PACS number: 43.50.Qp [doi:10.1250/ast.27.309]

\section{Introduction}

The image of a car will be determined by various factors. The sound of the closing a car door may be one of the determining factors. Much effort has been made to improve the quality of this sound. In this study, the relation between the subjective impression of this sound and the mental image of the car was examined in an experiment using German and Japanese participants.

\section{Experiment}

\subsection{Stimuli}

Eleven kinds of sound of the closing of passenger car doors were used as stimuli. They were recorded on a DAT tape using a dummy head (Head Acoustics, HMS I) at a point $85 \mathrm{~cm}$ from the door.

\subsection{Apparatus}

The sounds were reproduced with a DAT recorder and presented to the participants' ears through an amplifier, a free field equalizer and headphones (Beyer DT48) in a soundproof room. Similar equipment was used in the experiments in Germany and Japan.

\subsection{Procedure}

The experiment consisted of three parts. In Part 1 the subjective impression of the sounds was evaluated using semantic differential. The sound was presented three times with $1 \mathrm{sec}$ intervals and participants were instructed to evaluate the impression of the sound using semantic differential. Fifteen pairs of adjective scales were selected on the basis of our former studies [1,2]. They are 7-point scales and are listed in Table 1. The adjective scales were presented one after the other on a monitor of a computer in random order. Participants evaluated the impression and responded using a computer keyboard. They were informed that the sounds were from the closing of car doors. The experiment was conducted after training using two sounds which were not used in the experiment. In Part 2, after listening to the sound again, participants selected the appropriate image of the car. They were given a list of types of car. There were five categories:

\footnotetext{
*e-mail: kuwano@env.eng.osaka-u.ac.jp
}

luxurious sedan, expensive sporty car, economic sedan, pickup truck and another category of their own description. The participants were also asked to guess the name of the model of the car from the sound. In Part 3 the same sound was presented again and the impression was evaluated using semantic differential, as in Part 1. The three parts were conducted successively.

2.4. Participants

2.4.1. Experiment in Germany

Three females and seventeen males with normal hearing ability, aged between 26 and 57 (mean age 33.3), participated in the experiment in Germany. All the participants were German and except for the two had their own cars.

\subsubsection{Experiment in Japan}

Three females and seventeen males with normal hearing ability, aged between 20 and 40 (mean age 24.8), participated in the experiment in Japan. All the participants were Japanese and all of them had experience of driving cars. All the participants except for two had their own cars or drove a family car.

\section{Results}

High coefficients of correlation between the results of Part 1 and Part 3 were found with the German group ( $r=$ $0.941)$ and the Japanese group $(r=0.922)$. This suggests that the formation of mental image in Part 2 has no effect on the evaluation of the sounds and that the evaluation of the participants is reliable. The results of Part 1 were used in the following analyses.

3.1. Cluster analysis of the sounds

The results of the cluster analysis of the sounds are shown in Figs. 1 and 2. The sounds were divided into three clusters for both groups of participants. Cluster 1 consists of Nos. 8 and 9 for the German group and Nos. 8, 9 and 11 for the Japanese group. Cluster 2 consists of Nos. 2, 3, 4, 5 and 6 for the German group and Nos. 2, 3, 5 and 6 for the Japanese group. Cluster 3 consists of Nos. 1, 7, 10 and 11 for the German group and Nos. 1, 4, 7 and 10 for the Japanese group. Similar results were found with German and Japanese groups of participants, except for sounds Nos. 4 and 11 . 
Table 1 Result of factor analysis (German result).

\begin{tabular}{crrc}
\hline & I & II & III \\
\hline metallic-deep & 0.774 & -0.342 & 0.201 \\
heavy-light & -0.803 & 0.239 & 0.301 \\
dark-bright & -0.869 & 0.192 & -0.057 \\
sharp-dull & 0.740 & 0.091 & 0.404 \\
weak-powerful & 0.707 & -0.413 & -0.348 \\
shrill-calm & 0.667 & -0.385 & 0.378 \\
pleasant-unpleasant & -0.207 & 0.842 & -0.241 \\
beautiful-ugly & -0.238 & 0.838 & -0.178 \\
pleasing-unpleasing & -0.190 & 0.840 & -0.210 \\
pure-impure & -0.182 & 0.731 & 0.079 \\
noisy-quiet & 0.163 & -0.536 & 0.531 \\
loud-soft & -0.278 & -0.163 & 0.777 \\
hard-soft & 0.187 & -0.041 & 0.799 \\
gruff-gentle & 0.137 & -0.493 & 0.546 \\
rough-smooth & 0.478 & -0.356 & 0.502 \\
\hline
\end{tabular}

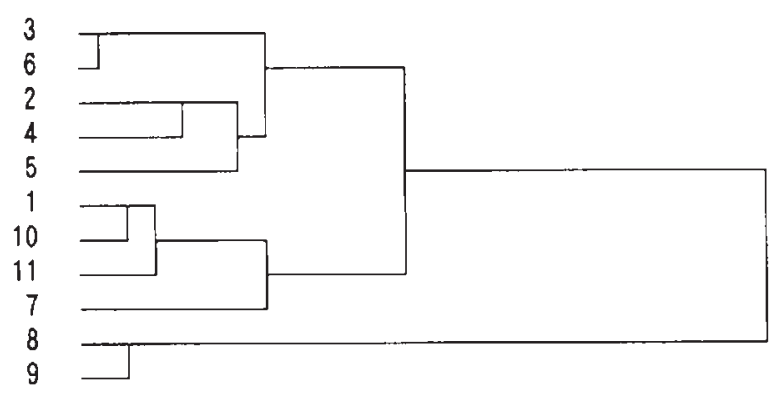

Fig. 1 Cluster analysis of German results.

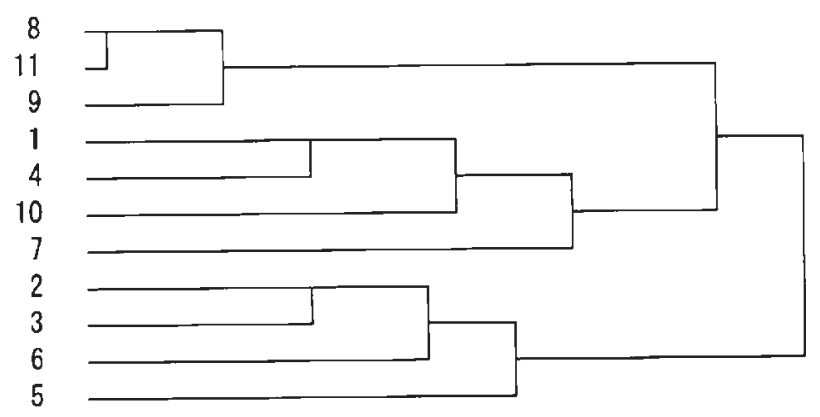

Fig. 2 Cluster analysis of Japanese results.

\subsection{Factor analysis of the adjective scales}

The results of the factor analysis of the adjective scales are shown in Tables 1 and 2. Three factors were extracted, as was usually found in our former studies [1,2], in both groups of participants though there is some difference. The three
Table 2 Result of factor analysis (Japanese result).

\begin{tabular}{cccc}
\hline & I & II & III \\
\hline pleasant-unpleasant & -0.828 & 0.281 & 0.123 \\
beautiful-ugly & -0.823 & 0.231 & 0.134 \\
pleasing-unpleasing & -0.825 & 0.115 & 0.058 \\
rough-smooth & 0.792 & 0.321 & 0.038 \\
noisy-quiet & 0.779 & 0.222 & 0.277 \\
gruff-gentle & 0.727 & 0.327 & 0.178 \\
hard-soft & 0.652 & 0.453 & 0.140 \\
metallic-deep & 0.389 & 0.652 & -0.387 \\
dark-bright & 0.095 & -0.748 & 0.373 \\
sharp-dull & 0.134 & 0.788 & -0.236 \\
pure-impure & -0.407 & 0.750 & -0.078 \\
shrill-calm & 0.485 & 0.674 & -0.253 \\
loud-soft & 0.242 & -0.208 & 0.781 \\
weak-powerful & 0.083 & 0.297 & -0.801 \\
heavy-light & -0.058 & -0.625 & 0.640 \\
\hline
\end{tabular}

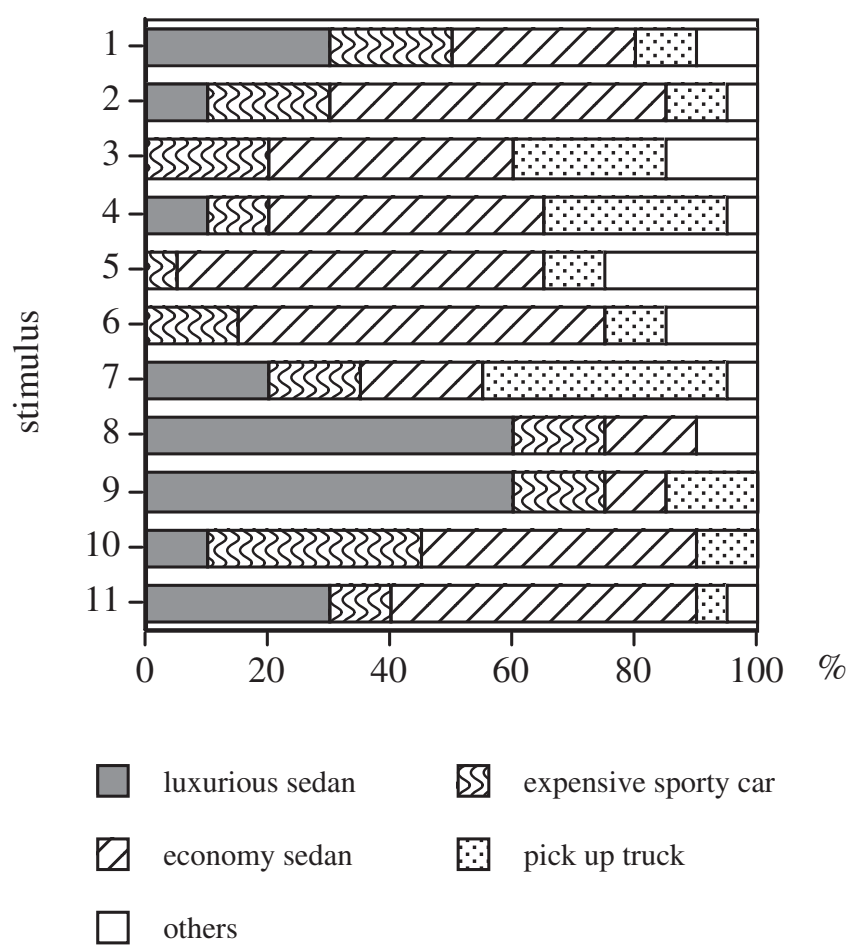

Fig. 3 Result of Part 2 by German participants.

factors can be interpreted as "pleasant factor," "metallic factor" and "powerful/hard factor."

3.3. Mental image from the sounds

The image of the car was estimated using the sounds in Part 2. The results are shown in Figs. 3 and 4. 


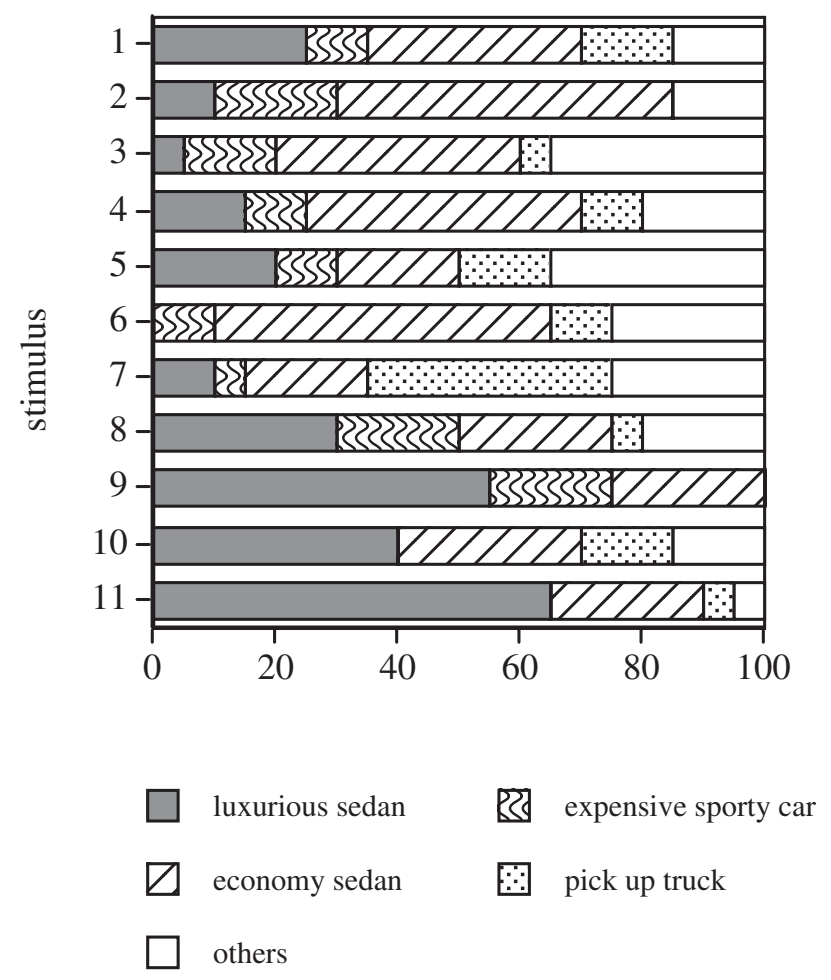

Fig. 4 Result of Part 2 by Japanese participants.

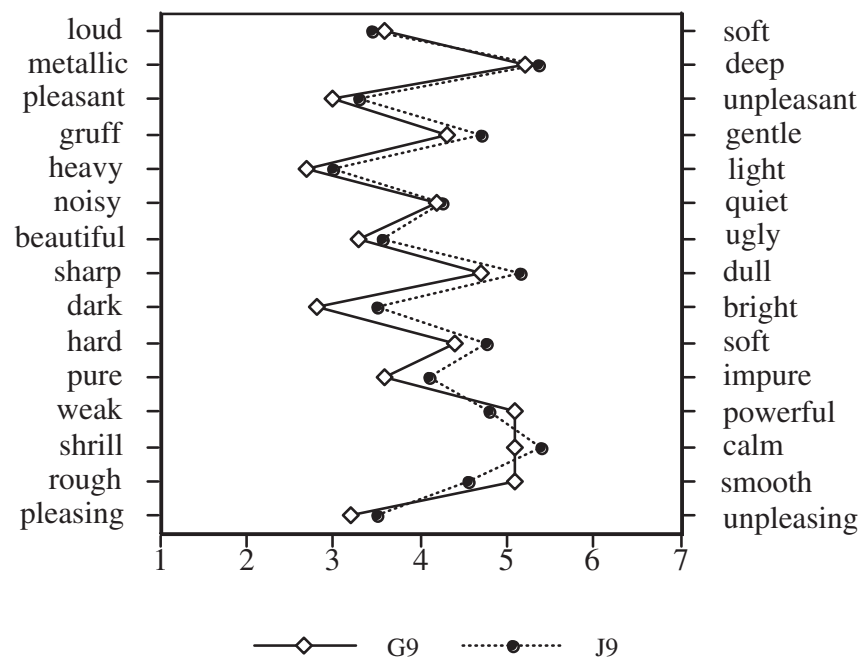

Fig. 5 Profiles of the sound No. 9 that belongs to cluster 1. Open diamonds and filled circles show the results with German and Japanese participants, respectively. The coefficient of correlation between German and Japanese results was 0.935 .

\section{Discussion}

Examples of the profiles of each cluster group are shown in Figs. 5-7. Generally speaking, high correlation was found between the German and Japanese results. The profiles of sound No. 9, shown in Fig. 5, indicate that this sound was perceived as being deep, pleasant, gentle and heavy. This suggests that cluster 1 is a group of pleasant sounds. The images from the sounds which belong to cluster 1 were associated with luxurious sedan by high percentages as shown

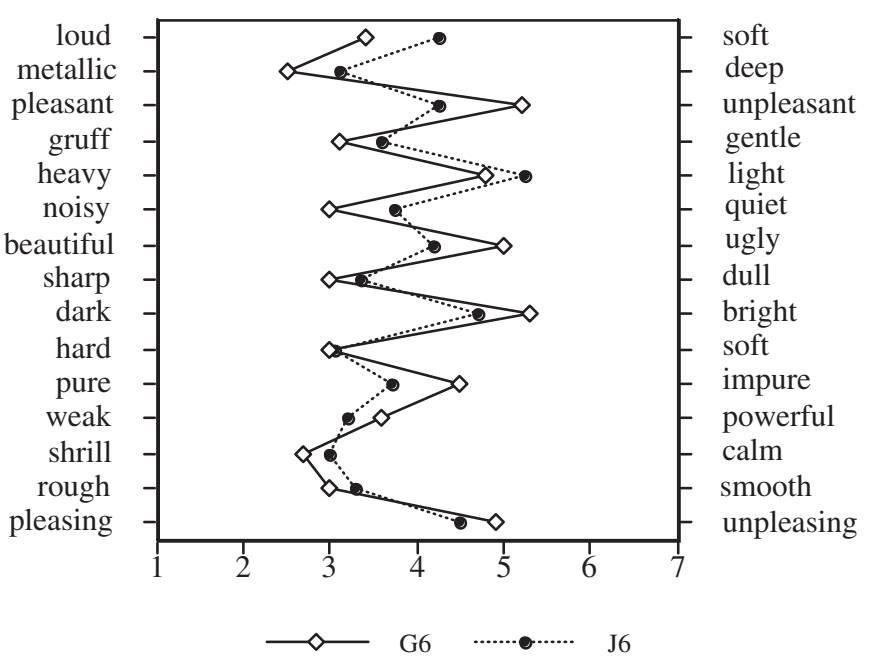

Fig. 6 Profiles of the sound No. 6 that belongs to cluster 2. Open diamonds and filled circles show the results with German and Japanese participants, respectively. The coefficient of correlation between German and Japanese results was 0.810 .

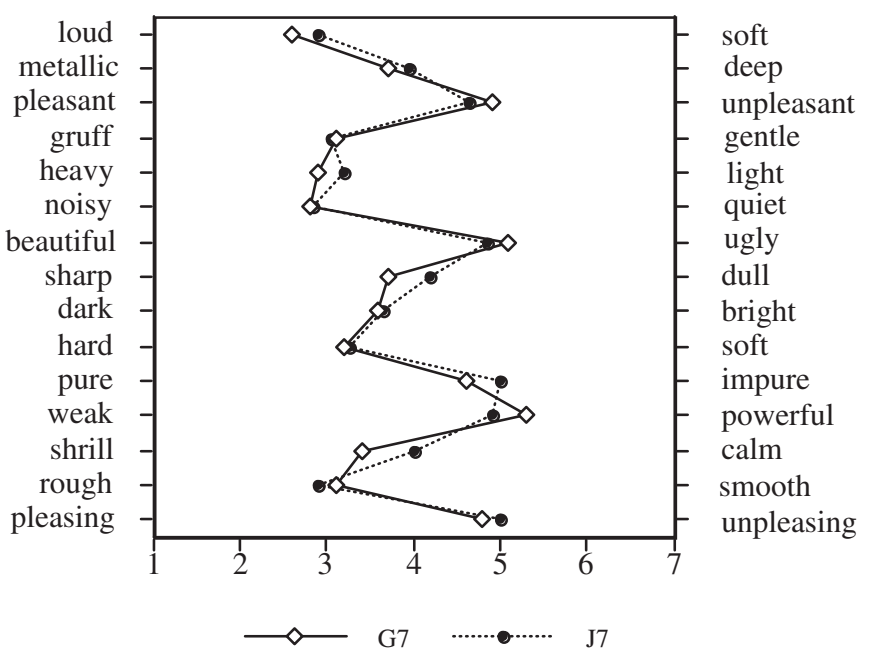

Fig. 7 Profiles of the sound No. 7 that belongs to cluster 3. Open diamonds and filled circles show the results with German and Japanese participants, respectively. The coefficient of correlation between German and Japanese results was 0.946 .

in Figs. 3 and 4. On the other hand, the profiles of sound No. 6, shown in Fig. 6, indicate that this sound was perceived as being metallic, unpleasant, gruff and light. This suggests that cluster 2 is a group of unpleasant sounds. The images of the sounds which belong to cluster 2 were associated with economy sedan by high percentages. The profiles of sound No. 7, shown in Fig. 7, indicate that this sound was perceived as being unpleasant, gruff, powerful and rough. This sound seems to have conveyed different impression from the other sounds. The image associated with this sound was of a pickup truck by high percentages in both groups of participants. The sounds which belong to cluster 3 seem to produce different images from the sounds of the other two clusters. 


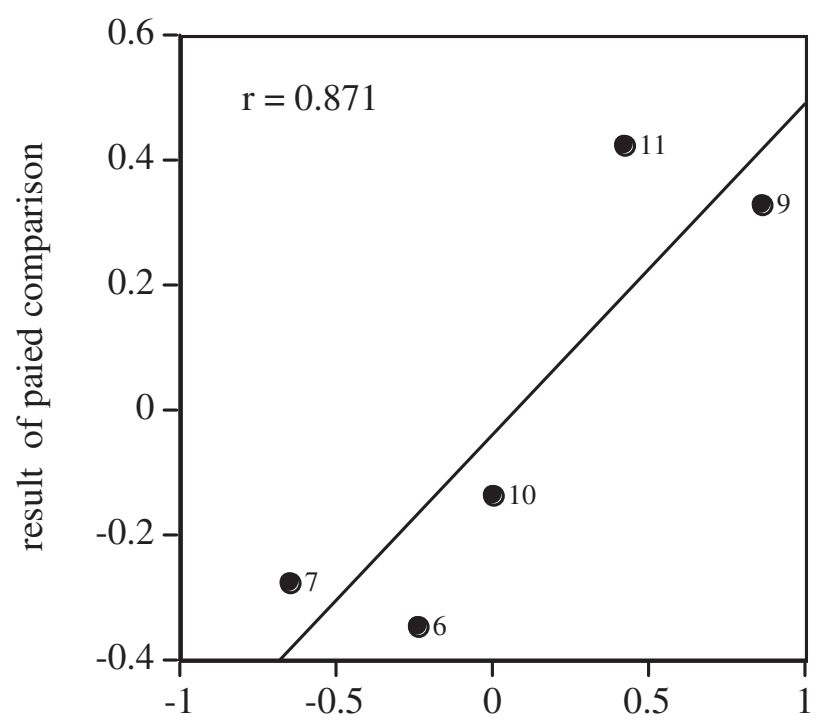

factor score (pleasant)

Fig. 8 The relation between the results of semantic differential (factor score of pleasantness) and those of paired comparison.

Sound No. 10 was estimated as the sound from an expensive sporty car by German participants.

A supplementary experiment was conducted using the five sounds from 11 sounds used in the experiment. They were stimuli Nos. 6, 7, 9, 10 and 11. Nine male and 79 female Japanese university students were asked to judge the pleasant impression using paired comparison. The sounds were presented in a lecture hall via a loudspeaker. The relation between the results of semantic differential (factor score of pleasantness) and those of paired comparison is shown in Fig. 8. Fairly good correlation was found between them. The fact that similar results were found in the experiments in the wellcontrolled sound proof room and in a lecture hall suggests that the pleasant impression of the sound of a car door closing is stable. This gives us a hint to find the factors determining the impression of the sound quality.
Another interesting feature is the relation between the evaluation of the car door sounds and the image of the brand names $[3,4]$. It was found that German participants selected world-famous brand-names of German cars as luxurious sedans brands. According to a survey by the German automobile association (ADAC), the selected brands represent the leading German manufacturers of luxury automobiles. Hence, it is rather likely that the image of the respective brand may have influenced the names given in Part 2.

\section{Final remarks}

The results from the German and Japanese groups of participants showed fairly good agreement, though a slight difference was found with some sounds. It was found that the quality of the sound of a car door closing was perceived differently in different cases. A pleasant impression of the sound seems to be related to the qualities, gentle, deep and heavy. It was suggested that the image of a car is related to the sound produced by the door of the car. Since our hearing is sensitive to the temporal change in a short period [5], it would be important to do further analysis taking temporal factors of the sounds into consideration.

\section{References}

[1] S. Namba, S. Kuwano, K. Kinoshita and K. Kurakata, "Loudness and timbre of broad-band noise mixed with frequency modulated sounds," J. Acoust. Soc. Jpn. (E), 13, 229-232 (1992).

[2] S. Namba, S. Kuwano and M. Koyasu, "The measurement of temporal stream of hearing by continuous judgments - in the case of the evaluation of helicopter noise," J. Acoust. Soc. Jpn. (E), 14, 341-352 (1993).

[3] Th. Filippou, H. Fastl, S. Kuwano, S. Namba, S. Nakamura and H. Uchida, "Door sound and image of cars," Fortschritte der Akustik, DAGA 2003, Verl.: Dt. Gesell. für Akustik e. V., Oldenburg, pp. 306-307 (2003).

[4] H. Fastl, "Psychoacoustics and sound quality," in Communication Acoustics, J. Blauert, Ed. (Springer-Verlag, Berlin, Heidelberg, 2005), pp. 139-162.

[5] S. Kuwano, S. Namba, K. Ohta, H. Takeda, K. Sato and Y. Tsuji, "Relation between envelope pattern and sound quality of impulsive sounds," J. Acoust. Soc. Jpn. (E), 20, 153-155 (1999). 